\title{
Surfer's Myelopathy : Case Series and Literature Review
}

\author{
June Ho Choi, M.D., Jung-Ki Ha, M.D., ${ }^{2}$ Chung Hwan Kim, M.D., Ph.D., ${ }^{2}$ Jin Hoon Park, M.D., Ph.D. \\ Department of Neurological Surgery, Asan Medical Center, University of Ulsan College of Medicine, Seoul, Korea \\ Department of Orthopaedic Surgery, ${ }^{2}$ Gangneung Asan Hospital, University of Ulsan College of Medicine, Gangneung, Korea
}

Three male patients diagnosed with surfer's myelopathy (19-30 years) were admitted to our hospital. All three patients were novice surfers showing a typical clinical course of rapid progression of paraplegia following the onset of back pain. Typical history and magnetic resonance imaging features indicated the diagnosis of surfer's myelopathy. Two patients received high-dose steroid therapy and the other was treated with induced hypertension. One patient treated with induced hypertension showed almost full recovery; however, two patients who received high-dose steroid therapy remained completely paraplegic and required catheterization for bladder and bowel dysfunction despite months of rehabilitation. Our case series demonstrates the potentially devastating neurological outcome of surfer's myelopathy; however, early recovery in the initial 24-72 hours of presentation can occur in some patients, which is in accordance with previous reports. Ischemic insult to the spinal cord is thought to play a crucial role in the pathophysiology of surfer's myelopathy. Treatment recommendations include hydration, induced hypertension, early spinal angiography with intra-arterial intervention, intravenous tissue plasminogen activator, and high-dose steroid therapy; however, there is no standardized treatment option available. Early recovery appears to be important for long-term neurological outcome. Induced hypertension for initial treatment can be helpful for improving spinal cord perfusion; therefore, it is important for early and long-term neurological recovery. Education and awareness are essential for preventing surfer's myelopathy and avoiding further deterioration of neurological function.

Key Words : Water sports · Spinal cord injuries.

\section{INTRODUCTION}

Surfer's myelopathy is a rare nontraumatic spinal cord injury usually affecting novice surfers. Ischemic insult is thought to play a crucial role in the pathophysiology of this rare disease $^{29)}$. Surfing has become a popular sport in South Korea. Because more people are attempting to learn surfing, increased numbers of patients with surfer's myelopathy are anticipated. We encountered three cases of complete paraplegia resulting from surfer's myelopathy. All three patients were novice surfers. One patient showed almost full recovery, but the other two experienced complete paraplegia and bladder and bowel dysfunction despite months of rehabilitation. The purpose of this case series is to report on this rare disease and review the previous literature on this condition.

- Received : September 30, 2017 •Revised : February 11, 2018 •Accepted : March 5, 2018

- Address for reprints : Jin Hoon Park, M.D., Ph.D.

Department of Neurological Surgery, Gangneung Asan Hospital, University of Ulsan College of Medicine, 38 Bangdong-gil, Sacheon-myeon, Gangneung 25440 , Korea Tel : +82-33-610-3260, Fax : +82-33-610-4960, E-mail : grandblue@gnah.co.kr

This is an Open Access article distributed under the terms of the Creative Commons Attribution Non-Commercial License (http://creativecommons.org/licenses/by-nc/4.0) which permits unrestricted non-commercial use, distribution, and reproduction in any medium, provided the original work is properly cited. 


\section{CASE REPORT}

\section{Case 1}

A 30-year-old Korean male $(73 \mathrm{~kg} ; 173 \mathrm{~cm}$; body mass index [BMI], 24.39) felt progressive and rapid loss of leg strength after his first surfing lesson. He had previously felt some back pain, which was aggravated while he was practicing standing up quickly from the prone position for several hours. He felt weakness in both legs and came to shore, discovering that he could barely walk. He was completely paraplegic below the level of T11 upon presentation to emergency room (ER). Magnetic resonance imaging (MRI) findings revealed hyperintense anterior central cord signal on T2-weighted imaging from T7 to the conus (Fig. 1). Diffusion tensor imaging showed mildly increased signal on diffusion-weighted imaging with apparent diffusion coefficient decrease from T9 to T12. He had no past history or recent illness, signs, or symptoms of infectious disease or previous back pain. Laboratory tests were normal and cerebrospinal fluid (CSF) analysis showed mild pleocytosis (red blood cell, 195; white blood cell, 50; neutrophil count, 92\%). He was diagnosed with surfer's myelopathy, given the obvious clinical history and imaging findings, and he was treated with pulse steroid therapy ( $1 \mathrm{~g}$ of methylprednisolone for 3 days and tapering out with prednisolone thereafter) and rehabilitation. Despite the patient's efforts, there was no recovery of motor strength in the bilateral lower extremities and bladder dysfunction.

\section{Case 2}

A 19-year-old healthy Korean male $(93 \mathrm{~kg} ; 175 \mathrm{~cm}$; BMI, 30.36) experienced rapid onset of acute back pain with gradual sensory loss and ongoing weakness in the lower extremities when returning to shore from his first surfing lesson. Though he had fallen off the board into the water once, there was no impact trauma. First, he developed acute, severe pain in his back with a tingling sensation in his lower extremities and progressive weakness within half an hour. He was taken to the local emergency room, where he presented with complete paraplegia with absence of bladder function. He decided to transfer to our hospital. Physical evaluation showed that his lower extremity strength was $3 / 3$. Both paresthesia and hypoesthesia below the level of the umbilicus were noted. Proprioception of the big toes was intact. Perianal sensation was de- creased and urinary retention was present. Bulbocavernosus reflex was intact. He underwent MRI of the thoracic and lumbar spine without contrast, which showed increased T2 signal intensity in the gray matter of the central spinal cord from T10 to the conus (Fig. 2); otherwise, MRI was negative for structural lesions including disc herniation, fractures, spondylosis, or vascular lesions. Laboratory tests were within normal limits. Considering his clinical history as a first time surfer, the patient was suspected of having surfer's myelopathy. He was admitted to the department of neurosurgery, and we focused on maintaining the degree of hypertension (average mean arterial blood pressure $>85 \mathrm{mmHg}$ ) and did not administer steroids. His motor grade of the lower extremities recovered to

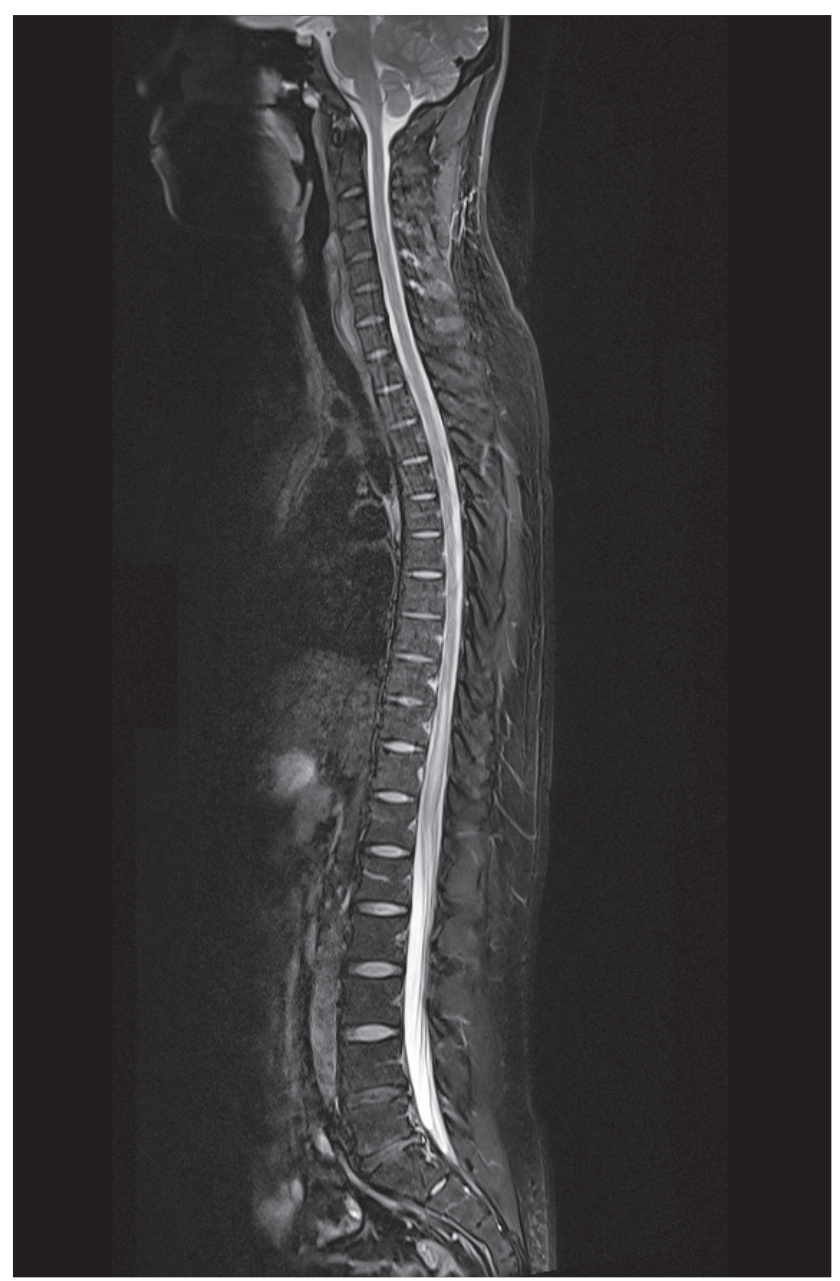

Fig. 1. Case 1 : a 30-year-old Korean male. Midline sagittal T2-weighted MRI of the whole spine was obtained at the ER. MRI findings revealed hyperintense signals of the anterior central cord on T2-weighted imaging from $\mathrm{T7}$ to the conus. MRI : magnetic resonance imaging, ER : emergency room. 
$4+/ 4+$, and he was able to ambulate with assistance the day after admission; however, there was little improvement in paresthesia and hypoesthesia. The next day, he transferred to his hometown hospital to be close to his parents. One month after the event, he recovered his strength to almost normal levels with intact bladder function and improved sensory deficit.

\section{Case 3}

A 21-year-old Korean male with no medical history had a first time surfing lesson during which he felt a sudden cramping pain in his lower back. A few minutes later, he developed a tingling sensation of the lower extremities followed by progressive motor weakness. He visited the local ER, where he

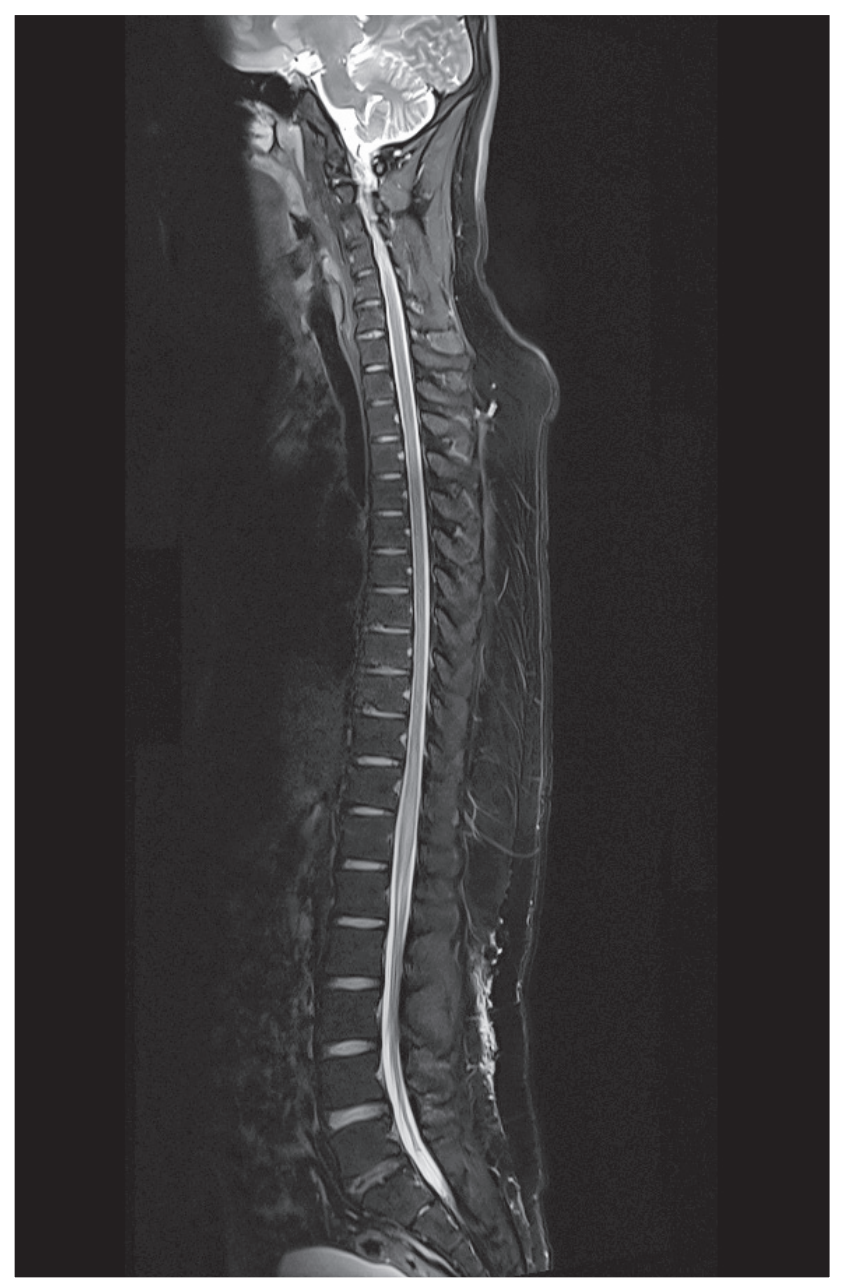

Fig. 2. Case 2 : a 19-year-old healthy Korean male. Midline sagittal T2weighted MRI of the whole spine was obtained at the ER. MRI showed increased T2 signal intensity in the gray matter of the central spinal cord from T10 to the conus. MRI : magnetic resonance imaging, ER : emergency room. was evaluated and diagnosed with complete spinal cord injury (American Spinal Injury Association class A) with absence of anal sphincter and bladder function. MRI of the lumbar spine without contrast showed only mild bulging of the L5-S1 disc and no other structural lesions that may develop acute paraplegia. He was transferred to our hospital for proper management and was admitted to the department of orthopedics. His initial neurologic examination showed no motor strength or sensation below the inguinal region. Laboratory tests showed mild leukocytosis, but there was no history or finding to suggest an infectious cause. Additional MRI of the thoracic spine with contrast was obtained, and diffused T2 prolongation of the central spinal cord from T10 to the conus medullaris was

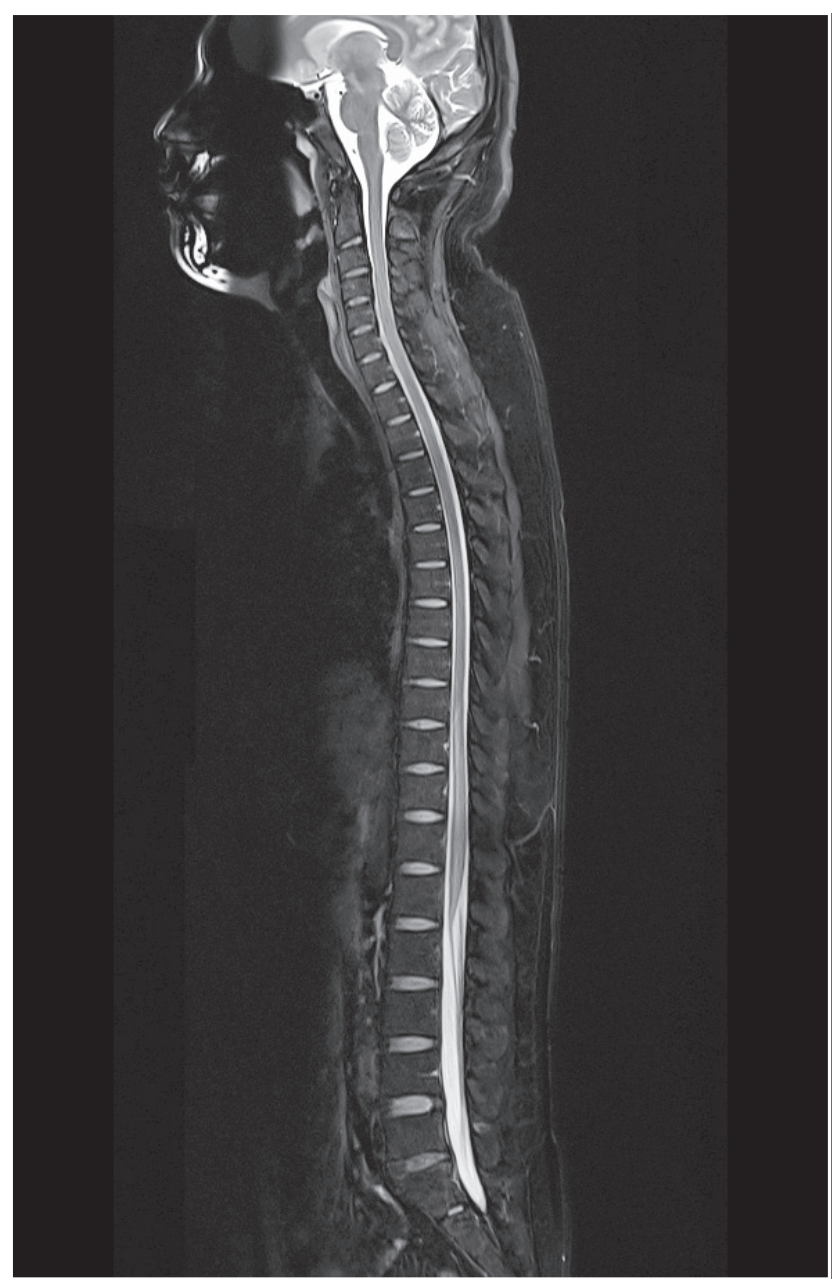

Fig. 3. Case 3 : a 21-year-old Korean male. Midline sagittal T2-weighted MRI of the whole spine was obtained at the ER. Increased signal and mild enlargement of the lower thoracic cord below the T10 vertebral level to the conus medullaris were observed. MRI : magnetic resonance imaging, ER: emergency room. 
noted (Fig. 3). He was treated with methylprednisolone based on the National Acute Spinal Cord Injury Study III (NASCIC III) protocol and underwent rehabilitation; however, motor strength of the lower extremities did not improved after 1 month of conservative care and rehabilitation. He recovered some degree of sensation but only when he was being pinched and pricked. Bladder dysfunction remained and catheterization was necessary until he was transferred to a local hospital for further rehabilitation.

\section{DISCUSSION}

As surfing has become one of the most popular leisure activities in South Korea, more people are trying to learn this exotic sport. Surfer's myelopathy is not related to a traumatic accident; it typically occurs during the first surf lesson wherein patients develop sudden acute back pain, spasms, or paresthesia. After the onset of back pain, patients usually develop new or progressive paresthesia and then paresis. When they visit the hospital (within a few hours after the first symptoms), their neurological deficit has reached its nadir. In addition to paresthesia and paresis, bowel and bladder dysfunction are also absent, which is immeasurably frustrating to this mostly healthy young patient population. All three patients in this report showed a typical course of surfer's myelopathy. The features of surfer's myelopathy are similar to those of acute spinal cord infarction. According to Robertson et al. ${ }^{24)}$, patients can recover from the ischemic insult, though not completely, despite the serious pathophysiology and disastrous deficits of acute spinal cord infarction, suggesting a chance for improved outcomes for surfer's myelopathy. This requires early recognition of signs and symptoms by the patient, which can be achieved by educating the public and surfing instructors, and emergent interventions for preventing deterioration following spinal cord injury.

In 2004, Thompson et al. ${ }^{29)}$ first described nine cases of this disease entity in eight men and one woman with an average age of 25 years (21-30 years); all nine patients were inexperienced surfers. They were commonly taking their first lesson and otherwise young and healthy without prior spinal or vascular problems. Other case reports and series were subsequently published after Thompson's introduction of this enti$\mathrm{ty}^{1,2,7-9,12,16,18,19,22,26,28)}$. Thompson et al. ${ }^{29)}$ proposed this condition as an ischemic insult to the spinal cord; therefore, they tried triple $\mathrm{H}$ therapy (hypertension, hypervolemia, and hemodilution) and high-dose steroids in some cases to improve outcomes. Though the reported outcomes usually turned out to be debilitating (patients needed wheelchairs to ambulate or required catheterization), some experienced good or full recovery within 24-72 hours of presentation ${ }^{13)}$. The patient in our report who was treated with induced hypertension also showed early recovery; therefore, this treatment appears to be crucial for good long-term neurologic outcomes. Though surfer's myelopathy appears to have devastating consequences, many cases show less severe deficits; therefore, it is diagnosed as a transient neurological event of unknown origin or otherwise not seeking medical attention ${ }^{13)}$.

Nakamoto et al. ${ }^{22)}$ published an article including 23 cases of surfer's myelopathy and analyzed the MRI features of the cases. All patients demonstrated T2 hyperintensities typically in the central portion of the spinal cord showing a "pencil-like" longitudinal extension from the midthoracic (T5-T10) level to the conus on sagittal images associated with cord swelling. Including the cases of this report, other reported cases and series to date have demonstrated no significant differences from the results in our study. They obtained 11 images with gadolinium enhancement and concluded that, while it can be helpful for the differential diagnosis, it does not contribute to the diagnosis of surfer's myelopathy. Diffusion-weighted images were obtained in several cases, and most (but not all) cases demonstrated restricted diffusion, thus it could be a good method for differentiating ischemic insult from other types of myelopathy, determining the approximate time of ongoing ischemia, and predicting disease prognosis ${ }^{20,30)}$ but it does not enhance diagnostic workup $^{13)}$.

Given the location of the injury on imaging studies and the anatomical characteristics of the midthoracic region, surfer's myelopathy is considered an ischemic injury of the thoracic spinal cord, and prolonged prone hyperextension has been suggested as the primary cause $\mathrm{e}^{1,2,7-9,12,13,16,18,19,22,26,28,29)}$. The continuous prone hyperextended posture while paddling can possibly cause avulsion of perforating vessels, vasospasm of the artery of Adamkiewicz, or transient ischemia in areas of borderline perfusion as a result of spinal cord tension due to hyperextension. Prolonged spinal hyperextension has been previously reported as a possible mechanism for spinal cord injury $^{23)}$. Other possible mechanisms have been proposed, in- 
cluding venous infarction from obstruction of the inferior vena cava obstruction by the liver while lying prone over the surfboard, or fibrocartilaginous embolism, which is potentially triggered by the Valsalva maneuver while standing up on the surfboard ${ }^{2,8,9,26)}$.

Since surfer's myelopathy is considered a result of acute ischemic insult (which is caused by occlusion, spasm, external compression, or avulsion), Freedman et al. ${ }^{13)}$ recommend that spinal angiography should be considered in the acute evaluation and management of surfer's myelopathy. Spinal angiography can find underlying vascular anomalies or anatomical variations that are vulnerable to ischemic insult or confirm intravascular mechanisms disrupting spinal cord perfusion. Moreover, it allows direct intra-arterial intervention (i.e., delivering tissue plasminogen activator [ $\mathrm{tPA}]$ or nimodipine) ${ }^{13}$. Nevertheless, spinal angiography has been used infrequently in previous reports, which is possibly due to the inherent risk and limited availability of spinal angiography as well as the decision of a physician who is not familiar with this disease.

As surfer's myelopathy usually happens to novice surfers, it is important to educate beginners as well as instructors, considering its debilitating outcome. In their case report, Karabegovic et al. ${ }^{16)}$ introduced Surfer's Myelopathy Foundation tips for safe surfing. The tips were issued under the mnemonic "SPINE" (S : sit on your board while waiting for waves; P : pace your time in the water [30-minute limit]; I : insist on a knowledgeable surfing instructor; $\mathrm{N}$ : notice signs of pain and discomfort in your back; $\mathrm{E}$ : exit the water and seek medical attention if you experience pain, tingling, or weakness) ${ }^{27}$. Freedman et al. ${ }^{13)}$ also recommend that individuals flying long distances should rest and rehydrate before engaging in surfing lessons as they speculated that an alteration in rheology (dehydration from long-haul flight) may play a role in this event.

There are no standardized treatment options for this rare disease as there is no strong evidence or controlled studies due to its rarity. Freedman et al. ${ }^{13)}$ shared their treatment recommendations based on case reports, expert opinions, and their experiences. They recommended that, when available, emergent spinal angiography should be considered with administration of intra-arterial tPA in the presence of confirmed embolic occlusion or nicardipine in the presence of vasospasm. They recommended considering intravenous $\mathrm{PA}$ in case there is no identified occlusion or emergent spinal angiography is not available. Lumbar drain placement can also be considered as it allows CSF sampling (which can help rule out other etiologies of acute atraumatic spinal cord injury) and reduces CSF pressures to $10-15 \mathrm{mmHg}$ (which showed a neuroprotective effect from previous reports of thoracic surgery and acute spinal cord injury $)^{3,10,17,31)}$. Elevating mean arterial pressure (MAP) over $85 \mathrm{mmHg}$ for at least 24 hours was also recommended $^{133}$. Hawryluk et al. ${ }^{14)}$ demonstrated that elevating average MAP over $85 \mathrm{mmHg}$ for the initial 5-7 days of acute spinal cord injury has shown better outcome. The most recent American Association of Neurological Surgeons/Congress of Neurological Surgeons (AANS/CNS) guidelines for spinal cord injury also recommend maintaining MAP between 85 and $90 \mathrm{mmHg}$ for the first 7 days following an acute spinal cord injury $^{25)}$. In our case series, the patient showing early recovery and good long-term neurologic outcome was treated with induced hypertension, which can be achieved initially with intravenous fluids, careful use of narcotics for pain, and vasopressors ${ }^{13)}$. It has been reported that reducing CSF pressure and increasing MAP can synergistically improve spinal cord perfusion ${ }^{4,17,21,31)}$. High-dose steroid therapy was frequently used in previous reports in the context of acute spinal cord injury in consideration of the NASCIS III methylprednisolone protocol ${ }^{5,6)}$. Freedman et al. ${ }^{13)}$ asserted that patients with this condition can be appropriate candidates for steroid therapy as surfer's myelopathy mostly affects healthy and young patients without trauma. But until now, steroid therapy has not been studied for nontraumatic ischemic spinal cord injuries. Given the questionable benefits of high-dose steroid therapy combined with high rates of adverse effects, the most recent AANS/CNS guidelines changed their recommendations for steroid treatment in spinal cord injury ${ }^{15)}$; however, those guideline changes are still controversial ${ }^{11}$.

\section{CONCLUSION}

Surfing has become a popular sport in South Korea. The etiology of surfer's myelopathy remains unknown, but ischemic insult to the spinal cord is considered to play a role. Though this condition shows rapid progression of neurologic deterioration and severe deficits, some patients experience good or full recovery within 24-72 hours of presentation. Therefore, surfers and instructors should be educated on detecting the early signs and symptoms of this condition, as 
these interventions are necessary to prevent further deterioration. Hydration, induced hypertension, early spinal angiography with intra-arterial intervention, intravenous tPA, and high-dose steroid therapy are considered acute treatments, but there is still a lack of data for establishing standardized treatments. As seen in this and previous reports, early recovery is important for good long-term neurological outcome. According to the AANS/CNS guidelines, elevating MAP can be helpful in improving spinal cord perfusion; therefore, it is important for early and long-term neurological recovery.

\section{CONFLICTS OF INTEREST}

No potential conflict of interest relevant to this article was reported.

\section{INFORMED CONSENT}

Informed consent was obtained from all individual participants included in this study.

\section{References}

1. Aoki M, Moriizumi S, Toki M, Murakami T, Ishiai S : Rehabilitation and long-term course of nontraumatic myelopathy associated with surfing. Am J Phys Med Rehabil 92 : 828-832, 2013

2. Avilés-Hernández I, García-Zozaya I, DeVillasante JM : Nontraumatic myelopathy associated with surfing. J Spinal Cord Med 30 : 288-293, 2007

3. Bilal $H, O^{\prime}$ Neill $B$, Mahmood S, Waterworth $P$ : Is cerebrospinal fluid drainage of benefit to neuroprotection in patients undergoing surgery on the descending thoracic aorta or thoracoabdominal aorta? Interact Cardiovasc Thorac Surg 15 : 702-708, 2012

4. Bobadilla JL, Wynn M, Tefera G, Acher CW : Low incidence of paraplegia after thoracic endovascular aneurysm repair with proactive spinal cord protective protocols. J Vasc Surg 57 : 1537-1542, 2013

5. Bracken MB, Shepard MJ, Holford TR, Leo-Summers L, Aldrich EF, Fazl $M$, et al. : Administration of methylprednisolone for 24 or 48 hours or tirilazad mesylate for 48 hours in the treatment of acute spinal cord injury. results of the third national acute spinal cord injury randomized controlled trial. National Acute Spinal Cord Injury Study. JAMA 277 : 1597-1604, 1997

6. Bracken MB, Shepard MJ, Holford TR, Leo-Summers L, Aldrich EF, Fazl
M, et al. : Methylprednisolone or tirilazad mesylate administration after acute spinal cord injury: 1-year follow up. results of the third national acute spinal cord injury randomized controlled trial. J Neurosurg 89 : 699-706, 1998

7. Chang CW, Donovan DJ, Liem LK, O'Phelan KH, Green DM, Bassin S, et al. : Surfers' myelopathy: a case series of 19 novice surfers with nontraumatic myelopathy. Neurology 79 : 2171-2176, 2012

8. Chung HY, Sun SF, Wang JL, Lai PH, Hwang CW : Non-traumatic anterior spinal cord infarction in a novice surfer: a case report. J Neurol Sci 302 : 118-120, 2011

9. Dhaliwal PP, Cenic A, Eesa M, du Plessis $S$ : An unusual case of myelopathy: surfer's myelopathy. Can J Neurol Sci 38 : 354-356, 2011

10. Estrera AL, Sheinbaum R, Miller CC, Azizzadeh A, Walkes JC, Lee TY, et al. : Cerebrospinal fluid drainage during thoracic aortic repair: safety and current management. Ann Thorac Surg 88 : 9-15; discussion 15, 2009

11. Fehlings MG, Wilson JR, Cho $\mathrm{N}$ : Methylprednisolone for the treatment of acute spinal cord injury: counterpoint. Neurosurgery 61 Suppl 1 : 36-42, 2014

12. Fessa CK, Lee BS : An Australian case of surfer's myelopathy. Clin J Sport Med 22 : 281-283, 2012

13. Freedman BA, Malone DG, Rasmussen PA, Cage JM, Benzel EC : Surfer's myelopathy: a rare form of spinal cord infarction in novice surfers: a systematic review. Neurosurgery $78:$ 602-611, 2016

14. Hawryluk G, Whetstone W, Saigal R, Ferguson A, Talbott J, Bresnahan J, et al. : Mean arterial blood pressure correlates with neurological recovery after human spinal cord injury: analysis of high frequency physiologic data. J Neurotrauma 32 : 1958-1967, 2015

15. Hurlbert RJ, Hadley MN, Walters BC, Aarabi B, Dhall SS, Gelb DE, et al. : Pharmacological therapy for acute spinal cord injury. Neurosurgery 76 Suppl 1 : S71-S83, 2015

16. Karabegovic A, Strachan-Jackman S, Carr D : Surfer's myelopathy: case report and review. CJEM $13:$ 357-360, 2011

17. Kwon BK, Curt A, Belanger LM, Bernardo A, Chan D, Markez JA, et al. : Intrathecal pressure monitoring and cerebrospinal fluid drainage in acute spinal cord injury: a prospective randomized trial. J Neurosurg Spine $10: 181-193,2009$

18. Lieske J, Cameron B, Drinkwine B, Goretzke S, Alemi L, Needham K, et al. : Surfer's myelopathy-demonstrated by diffusion-weighted magnetic resonance imaging: a case report and literature review. J Comput Assist Tomogr 35 : 492-494, 2011

19. Lin CY, Fu JH, Li SC, Lai PH : Surfer's myelopathy. QJM 105 : 373-374, 2012

20. Loher TJ, Bassetti CL, Lövblad KO, Stepper FP, Sturzenegger M, Kiefer C, et al. : Diffusion-weighted MRI in acute spinal cord ischaemia. Neuroradiology 45 : 557-561, 2003

21. Martirosyan NL, Kalani MY, Bichard WD, Baaj AA, Gonzalez LF, Preul $M C$, et al. : Cerebrospinal fluid drainage and induced hypertension improve spinal cord perfusion after acute spinal cord injury in pigs. Neurosurgery 76 : 461-468; discussion 468-469, 2015

22. Nakamoto BK, Siu AM, Hashiba KA, Sinclair BT, Baker BJ, Gerber MS, 
et al. : Surfer's myelopathy: a radiologic study of 23 cases. AJNR Am J Neuroradiol 34 : 2393-2398, 2013

23. Rattihalli $R$, Khan $A$, Hussain $N$ : A reminder of the dangers of trampolining: spinal cord infarction secondary to hyperextension injury during trampolining. J Pediatr Neurosci 7 : 70-71, 2012

24. Robertson CE, Brown RD Jr, Wijdicks EF, Rabinstein AA : Recovery after spinal cord infarcts: long-term outcome in 115 patients. Neurology 78 : $114-121,2012$

25. Ryken TC, Hurlbert RJ, Hadley MN, Aarabi B, Dhall SS, Gelb DE, et al. : The acute cardiopulmonary management of patients with cervical spinal cord injuries. Neurosurgery 72 Suppl 2 : 84-92, 2013

26. Shuster $A$, Franchetto $A$ : Surfer's myelopathy--an unusual cause of acute spinal cord ischemia: a case report and review of the literature. Emerg Radiol 18 : 57-60, 2011
27. Surfer's Myelopathy Foundation : Tips for first time surfers. Available from : http://www.smawareness.org/what-is-sm/surfing-tips/

28. Takakura T, Yokoyama O, Sakuma F, Itoh R, Romero RR : Complete paraplegia resulting from surfer's myelopathy. Am J Phys Med Rehabil 92 : 833-837, 2013

29. Thompson TP, Pearce J, Chang G, Madamba J : Surfer's myelopathy. Spine (Phila Pa 1976) 29 : E353-E356, 2004

30. Thurnher MM, Bammer $R$ : Diffusion-weighted magnetic resonance imaging of the spine and spinal cord. Semin Roentgenol 41 : 294-311, 2006

31. Weigang E, Hartert M, von Samson P, Sircar R, Pitzer K, Genstorfer J, et al. : Thoracoabdominal aortic aneurysm repair: interplay of spinal cord protecting modalities. Eur J Vasc Endovasc Surg 30 : 624-631, 2005 